1 Centro Universitário Franciscano (Unifra) Santa Maria (RS), Brasil. cristiane.wagner@yahoo. com.br

2 Prefeitura Municipal de São José do Norte - São José do Norte (RS), Brasil. marianeperghersoares@ gmail.com

3 Prefeitura Municipal de Santa Maria - Santa Maria (RS), Brasil.

bskrebsky@gmail.com

4 Universidade Federal de Santa Maria (UFSM) -

Santa Maria (RS), Brasil.

unfer@terra.com.br

5 Universidade Federal de Santa Maria (UFSM) -

Santa Maria (RS), Brasil.

taisa.ferreira@gmail.com

\section{O processo de trabalho dos serviços de saúde frente a desastre de incêndio em casa noturna}

\author{
The work process of the health services regarding a nightclub fire \\ disaster
}

Cristiane Wagner ${ }^{\mathbf{1}}$, Mariane Pergher Soares ${ }^{\mathbf{2}}$, Bruna Skrebsky³, Beatriz Unfer ${ }^{\mathbf{4}}$, Taísa Gomes

Ferreira 5

RESUMO Este estudo qualitativo objetivou identificar e descrever a organização dos serviços de saúde e as estratégias de cuidado disponibilizadas às pessoas atingidas por um incêndio em uma casa noturna em Santa Maria (RS). Foram realizadas entrevistas semiestruturadas com trabalhadores de diferentes serviços de saúde no município. Identificaram-se fragilidades nos processos de referência e contrarreferência, comunicação e gestão, situações que existiam e se evidenciaram diante do cenário apresentado, dificultando a constituição de uma linha de cuidado. Pontos positivos foram observados, como a atuação do serviço de saúde mental e o estímulo à atuação multiprofissional, objetivando a integralidade do cuidado prestado às vítimas.

PALAVRAS-CHAVE Desastres. Serviços de saúde. Atenção Primária à Saúde. Incêndios.

ABSTRACT This qualitative study aimed to identify and describe the organization of health services and care strategies available for people affected by a fire in a nightclub in Santa Maria (RS). Semi-structured interviews were conducted with workers from different health services in the municipality. Fragilities were identified in the referral and counter-referral process, communication and management, situations that existed and were evident in the presented scenario, making it difficult to establish a care line. Positive points were observed, such as the performance of the mental health services and the stimulus to the multiprofessional practice, aiming the integrality of care provided to the victims.

KEYWORDS Disasters. Health services. Primary Health Care. Fires. 


\section{Introdução}

\section{O sistema de saúde brasileiro}

De acordo com a Constituição Federal Brasileira, a saúde é direito de todos e dever do Estado. Portanto, as ações e os serviços de saúde são de relevância pública, e o acesso é universal e igualitário, apoiado por políticas econômicas e sociais que visam reduzir os riscos de doenças e agravos (BRASIL, 1988).

Esse sistema não é composto apenas por ações assistenciais, mas, também, por serviços de vigilância em saúde, planejamento e gestão. As ações e os serviços de saúde são organizados em redes regionalizadas e hierarquizadas que abrangem promoção, proteção e recuperação da saúde, configurando o Sistema Único de Saúde (SUS). A gestão é realizada pela federação, pelos estados e municípios em suas competências específicas, com forte descentralização, permitindo a adoção de estratégias que considerem as especificidades e diversidades regionais que podem ser encontradas em um país com território amplo como o Brasil (BRASIL, 2011). Nesse contexto, o conceito de Redes de Atenção à Saúde (RAS), segundo Mendes (2010), pode ser entendido como sistemas organizados de prestação de serviços de saúde que servem a uma população definida e se responsabilizam pelos resultados clínicos, financeiros e sanitários relativos a essa população. Esses sistemas estão baseados no conceito de integração, um conceito amplo que inclui a noção de continuidade e coordenação do cuidado, gestão e comunicação fluida entre os serviços. Essas redes são compostas por serviços de atenção primária, secundária e terciária.

A Atenção Primária à Saúde (APS) é a porta de entrada preferencial do usuário no sistema e a coordenadora do cuidado na RAS, como estratégia para o cuidado integral da população. As Unidades Básicas de Saúde (UBS) desempenham um papel fundamental na garantia do acesso do usuário aos serviços de saúde e na longitudinalidade do cuidado. A Estratégia Saúde da Família (ESF), implantada em UBS, representa a reorientação do processo de trabalho como forma de expandir, qualificar e consolidar a atenção primária, por meio de equipes multiprofissionais responsáveis por um determinado território populacional. No Brasil, a cobertura da população pela ESF atinge cerca de $61 \%$, significando que a atenção primária é desenvolvida com dois modelos de atenção: o modelo tradicional e a ESF (BRASIL, 2012B). Em situações de emergências em saúde pública, a ESF pode fazer o reconhecimento territorial da sua população atingida, dar o suporte inicial para essa população e realizar a articulação com a rede de saúde (CARMO; PENNA; OLIVEIRA, 2008).

\section{Desastre}

Conforme a Política Nacional de Defesa Civil, os eventos adversos, naturais ou provocados, que causam danos humanos, materiais e ambientais em um local suscetível e prejuízos econômicos e sociais decorrentes desses eventos podem ser conceituados como desastres. Quando os desastres são provocados pela atuação do próprio homem, são denominados desastres humanos ou antropogênicos (BRASIL, 2007).

Os desastres podem provocar múltiplas vítimas e, na área da saúde, gerar desequilíbrio em um determinado período de tempo entre os recursos disponíveis e as necessidades das pesSoas (SÃO PAULO, 2012).

No ano de 2013, houve um incêndio em uma casa noturna em Santa Maria (RS), Brasil, que resultou em 242 vítimas fatais e centenas de sobreviventes. Comparado com ocorrências mundiais semelhantes, o incêndio ocorrido na cidade é o terceiro maior incêndio do mundo acontecido em uma casa noturna considerando-se o número de vítimas fatais, e o segundo maior em número de mortes no Brasil. Além das vítimas fatais, considera-se vítima toda pessoa que foi ou se 
sente atingida pelo incidente. Entre elas, estão os sobreviventes, os familiares e amigos dos mortos, os trabalhadores que atuaram tanto no resgate e no trabalho com os corpos quanto na atenção aos sobreviventes e a parcela da população do município que se sentiu afetada, direta ou indiretamente, pelo episódio.

Muitos sobreviventes sofreram queimaduras e inalaram fumaça tóxica, exigindo atendimento de emergência e de urgência. Juntamente aos danos físicos, ocorreram danos emocionais, dificuldades econômicas, além da necessidade de readaptação social. Em razão da magnitude do desastre na cidade, houve a necessidade de organizar os serviços de saúde de forma a estabelecer estratégias de cuidado e definir processos de trabalho específicos dirigidos à população afetada.

O objetivo deste trabalho foi identificar e descrever a organização dos serviços de saúde e as estratégias de cuidado na atenção às vítimas e analisar as percepções dos trabalhadores de saúde envolvidos no desastre em Santa Maria (RS), Brasil.

\section{Material e métodos}

Esta pesquisa consistiu em um estudo de abordagem qualitativa que utilizou a metodologia da pesquisa-ação, que, segundo Thiollent (2005), é um tipo de pesquisa social na qual há uma estreita associação com uma ação, aliando o conhecimento empírico ao científico, através da cooperação entre os participantes envolvidos. Essa metodologia foi escolhida por possibilitar aprimorar a prática, ao passo que se investiga a respeito dela (TRIPP, 2005).

De acordo com a Resolução do Conselho Nacional de Saúde $n^{\circ}$ 196/96, o projeto de pesquisa passou por avaliação de um Comitê de Ética em Pesquisa, sendo aprovado sob o registro CAAE (Certificado de Apresentação para Apreciação Ética) 23403313.0.0000.5346.

Para a coleta de dados, foram entrevistados trabalhadores de saúde dos serviços de saúde públicos assistenciais do município de Santa Maria (RS) que realizaram atendimento das vítimas. As entrevistas abertas semiestruturadas padronizadas seguiram roteiro que abordou as seguintes questões: quem foram as vítimas que o serviço acolheu; como o serviço respondeu às demandas dessas vítimas; como o entrevistado identificou as etapas percorridas no atendimento dessas pessoas; quais foram os sentimentos do entrevistado com relação ao trabalho desenvolvido na atenção às vítimas; como o entrevistado percebeu os serviços ofertados após o desastre; como o entrevistado descreve a circulação dos usuários no serviço; e se o entrevistado entende que existe comunicação entre os serviços e como ela ocorre. Além das entrevistas, também foram consultados dados de instituições governamentais como a Prefeitura Municipal de Santa Maria e o Ministério da Saúde.

A coleta de dados ocorreu no período de junho a novembro de 2013, em sala privativa, com o mínimo de interrupções. Participaram 17 trabalhadores: 2 médicos, 2 técnicos de enfermagem, 1 Agente Comunitário de Saúde (ACS), 10 enfermeiros, 1 assistente social e 1 fisioterapeuta. Os depoimentos foram gravados e transcritos literalmente.

\section{Resultados e discussão}

\section{Caracterização do município}

O município de Santa Maria localiza-se na região sul do Brasil, no centro do estado do Rio Grande do Sul (RS), e, segundo dados do Instituto Brasileiro de Geografia e Estatística (IBGE), tem uma população estimada em 260 mil habitantes (IBGE, 2010). A cidade possui 7 Instituições de Ensino Superior (IES), que recebem cerca de 30 mil estudantes universitários, vinculados a mais de 400 cursos de graduação e de pós-graduação. Possui o 
segundo maior contingente militar do País, com 19 organizações militares do Exército e um Esquadrão da Aeronáutica com seis unidades (SANTA MARIA, 2011), sendo caracterizada por ter uma população de muitos jovens cuja presença é transitória.

\section{Estrutura dos serviços de atenção à saúde}

Santa Maria pertence à região administrativa da $4^{\text {a }}$ Coordenadoria Regional de Saúde (4 ${ }^{\mathrm{a}}$ CRS), constituída por 32 municípios do estado do Rio Grande do Sul. A cobertura populacional de ACS é de $21 \%$ da população, e a ESF cobre apenas 19\% (BRASIL, 2012A). Conforme consulta ao Cadastro Nacional de Estabelecimentos de Saúde, no dia 24 de abril de 2013, e ao site da Prefeitura Municipal de Santa Maria (SANTA MARIA, C2017), os serviços da rede pública municipal de saúde em funcionamento no período do desastre consistiam em:

- Nível de Atenção Primário - 14 UBS com ESF; 14 Unidades Básicas de Saúde Tradicionais; 5 Unidades Básicas de Saúde Distritais.

- Nível de Atenção Secundário - 4 Centros de Apoio Psicossocial (Caps); 1 Centro de Referência em Saúde do Trabalhador (Cerest); 1 Centro de Testagem e Aconselhamento (Doenças Sexualmente Transmissíveis/Síndrome da Imunodeficiência Adquirida - DST/Aids); 1 Centro de Referência para Tuberculose; 1 Laboratório Municipal de Análises Clínicas; 1 Centro de Diagnóstico Especializado; 1 Centro Integrado da Saúde e Bem-Estar Social (Cisbes); 1 Centro de Especialidades Odontológicas (CEO).

- Nível de Atenção Terciário - 1 Hospital Municipal Casa de Saúde; 1 Hospital Universitário de Santa Maria (HUSM); 1 Pronto Atendimento Municipal; 1 Unidade de Pronto Atendimento 24h (UPA); 1
Serviço de Atendimento Móvel de Urgência (Samu).

\section{Organização do sistema de saúde durante e após o desastre}

\section{ATUAC̣ÃO EMERGENCIAL}

A atenção clínica e psicossocial, de caráter emergencial, forneceu assistência logo após o episódio e durante a primeira semana, trabalhando pela estabilização dos quadros clínicos e tendo suporte da rede de serviços e de profissionais existentes.

O cuidado emergencial clínico, nas primeiras 48 horas após o incêndio, foi dispensado pelos hospitais públicos e dois hospitais privados de Santa Maria e por dois centros especializados em queimados na capital do estado, Porto Alegre, situada a $291 \mathrm{~km}$. Foi prestada assistência às pessoas com queimaduras, intoxicação exógena e traumas físicos.

Para atender às demandas psicossociais, foi criado um serviço denominado 'Serviço de Acolhimento Psicossocial 24 horas'. Coordenado pela Secretaria Municipal de Saúde, instalou-se em anexo do Centro de Apoio Psicossocial, começando sua atuação no dia do desastre. Inicialmente, contou com a participação de profissionais voluntários para o atendimento aos familiares das vítimas e demais pessoas que se encontravam em sofrimento mental. Os atendimentos eram individuais e por telefone, com visitas domiciliares a sobreviventes, além de prestar apoio matricial na rede de atenção primária.

O Cerest empenhou-se na atuação com vistas à saúde do trabalhador, prestando atendimento àqueles indivíduos que foram afetados devido à sua atividade laboral, como trabalhadores da casa noturna, bombeiros, agentes funerários, policiais civis e militares, entre outros. Tal serviço também foi responsável por avaliar o local, dois dias após o desastre, e alertar os peritos que lá trabalhavam sobre os riscos de intoxicação tardia devido 
às substâncias tóxicas existentes no local.

Os serviços de saúde contaram com a cooperação da Força Nacional do SUS (FNSUS), de Organizações Não Governamentais (ONGs), como Cruz Vermelha e Médicos Sem Fronteiras (MSF), e de voluntários em geral para a estruturação de um sistema de apoio à população afetada direta ou indiretamente pelo desastre. Também houve auxílio de consultores do Ministério da Saúde e de profissionais articulados com a Política Nacional de Humanização (PNH).

\section{ATENC̣ÃO IMEDIATA}

Passou-se à organização da rede para o acompanhamento das vítimas. Foram criados o Acolhe Saúde (antes, denominado Serviço de Acolhimento 24 horas), com uma sede própria devido ao aumento da demanda, o Serviço de Matriciamento em Saúde Mental e o Centro Integrado de Atendimento a Vítimas de Acidentes (Ciava).

O Ciava foi criado nas dependências do Hospital Universitário de Santa Maria no mês seguinte ao do desastre, para responsabilizar-se pelo acompanhamento clínico das vítimas, disponibilizando exames, consultas especializadas e tratamentos para as complicações decorrentes da exposição à fumaça e a agentes químicos gerados pelo incêndio. O serviço foi pactuado com o Ministério da Saúde para fornecer acompanhamento das vítimas por um período de cinco anos, contando com a atuação de diversos núcleos profissionais, como dois técnicos farmacêuticos, quatro fisioterapeutas, dois enfermeiros, quatro técnicos em enfermagem, um assistente social, três psicólogos, dois médicos clínicos, um médico neurologista, um médico cirurgião plástico e cinco médicos psiquiatras. Esses profissionais foram contratados por processo seletivo emergencial. Já no primeiro mês após o desastre, 405 vítimas acessaram o serviço apresentando sintomas como ardência na garganta, cansaço, dispneia, rouquidão, irritação nos olhos, ardência e corrimento no nariz, além de relatos de insônia, ansiedade, tristeza e sensação de reviver o desastre (KIPPER; DALL'AGNOL; PAULESKI, 2014).

A Residência Multiprofissional Integrada da Universidade Federal de Santa Maria (UFSM) colaborou na assistência às vítimas atuando tanto nos serviços do município, nos quais os residentes já estavam inseridos, quanto nos novos serviços que surgiram a partir do desastre. Também houve integração da residência no que se refere à construção de fluxos e de comunicação entre os serviços nesse contexto, especialmente nos campos da saúde mental e da atenção primária, ressaltando-se o matriciamento de ambos.

Ainda que não vinculada à rede municipal de saúde, foi criada a Associação de Vítimas da Tragédia de Santa Maria (AVTSM), com o objetivo de unir os familiares e sobreviventes e prestar a assistência necessária por meio do trabalho voluntário de organizações como a Cruz Vermelha e a Associação Brasileira de Pesquisa e Ensino em Psicologia Transpessoal (Abrapet).

Em continuação, o Ministério da Saúde organizou o cadastramento das vítimas, a fim de possibilitar o acompanhamento longitudinal, por meio de um formulário eletrônico - FormSUS - disponível no site do Ministério ou pelo telefone da ouvidoria do SUS (BRASIL, C2017).

\section{CENÁRIO ATUAL}

A necessidade de comunicação entre os serviços demandou estratégias para seu aperfeiçoamento. Com esse intuito, reúnem-se semanalmente representantes dos serviços especializados que atuam na assistência às vítimas, representantes da AVTSM e membros da gestão em saúde do município, constituindo um Grupo Gestor do Cuidado.

\section{PROCESSO DE TRABALHO DOS PROFISSIONAIS DE SAÚDE}

Após a identificação das vítimas, foi necessário realizar a busca das pessoas que não 
haviam sido hospitalizadas, mas que tinham sido atingidas de alguma forma pelo desastre e que se encontravam dispersas. Por meio da atuação dos ACS, as pessoas foram contatadas; foram realizados o acolhimento, a avaliação de necessidades e o encaminhamento para tratamento médico ou para outros serviços, na medida de suas necessidades.

A identificação das vítimas foi um desafio para os serviços, uma vez que muitas pessoas não utilizavam habitualmente serviços públicos de saúde e os trabalhadores de saúde temiam ser invasivos. Os ACS eram orientados a conversar, oferecer os serviços das UBS e estimular a busca de cuidados, principalmente na área de saúde mental, mas quase ninguém acessou os serviços por esse motivo. Havia um sentimento de introspecção e de silêncio.

Com a criação de novos serviços e a organização dos já existentes para a atuação frente ao desastre, surgiram algumas questões relacionadas à atuação em rede. Cada serviço organizou-se conforme a sua demanda específica, sem articulação da gestão para que fosse uniforme e o trabalho se constituísse em redes. Essa falta de organização do fluxo dos usuários vítimas do desastre constitui um reflexo da desarticulação dos serviços de saúde no município. Um problema comum identificado nas entrevistas foi a comunicação. De 17 entrevistados, 7 relataram não existir comunicação entre os serviços, enquanto os demais ressaltaram que ela existe, mas com fragilidades.

Sobre o trabalho desenvolvido na atenção às vítimas, alguns trabalhadores consideraram que seu preparo na profissão facilitou a atuação no episódio, ainda que se sentissem sensibilizados com a situação, enquanto outros tiveram dificuldades e experimentaram sentimentos ambíguos. Houve sentimento de impotência e de empatia com as vítimas, alguns indicando também sentirem-se vítimas ao atuar no cuidado diante do desastre.

Nesse cenário, é importante considerar os dispositivos de apoio e de formação profissional na saúde. A atuação da Residência Multiprofissional, nos campos de práticas, foi evidente no que se refere à construção de fluxos e de comunicação entre os serviços, especialmente nos campos da saúde mental e da atenção primária.

Apesar das dificuldades encontradas na organização da rede após o desastre, alguns avanços são percebidos. Um dos grandes progressos foi a consolidação dos serviços de saúde mental. A partir da demanda gerada pelo desastre, o que vinha sendo construído no campo da saúde mental, como a implantação do matriciamento, por exemplo, encontrou o apoio e o incentivo necessários para a sua efetivação. Um trabalhador afirmou que, antes do desastre, não havia comunicação entre os Caps e as UBS, afirmando que não sabia nem o endereço, muito menos como era feita a distinção entre eles.

\section{DESAFIOS EM LONGO PRAZO}

Nos contextos mediato e imediato do desastre, foi necessário estruturar serviços emergenciais e de urgência, tanto clínicos como psicossociais. Entretanto, as vítimas necessitarão de acompanhamento em longo prazo, durante anos, pois mais demandas surgirão, cada uma apresentando uma especificidade, a seu tempo. Para isso, o sistema de saúde deve enfrentar os desafios para o cuidado longitudinal.

A longitudinalidade, considerada a característica central da APS, é entendida como o aporte regular de cuidados por parte da equipe de saúde, por seu uso consistente ao longo do tempo. O principal modo de realizar um cuidado longitudinal é a equipe conhecer o usuário inserido em seu contexto, suas características sociais, econômicas e culturais. Ele se desenvolve no momento em que a equipe de saúde serve como fonte de atenção contínua, independentemente de existirem ou não problemas específicos relacionados à saúde ou ao tipo de agravo, sendo estabelecida quando o usuário procura uma UBS e com ela constitui uma relação que 
perdura por um longo espaço de tempo. Assim, para a realização de um cuidado integral, deve-se partir do princípio de que a UBS de referência do paciente, no seu território, é quem deve ser a responsável por seu cuidado (STARFIELD, 2002).

$\mathrm{O}$ estabelecimento de vínculo entre os serviços e os usuários encontra obstáculos diante das características da população atingida, pois a maioria não reside no município. Esse desafio se intensifica no momento em que não está disponível um sistema de troca de informações entre os serviços de diferentes localidades. A criação de um prontuário eletrônico poderia auxiliar, pois possibilitaria, além da troca de informações, o controle e o aperfeiçoamento do processo de cuidado.

Os profissionais das UBS entendem que a centralização do serviço de saúde mental no Acolhe Saúde seria inadequada, pois o número de pessoas atingidas é muito grande, e o serviço não conseguiria assistir a toda a demanda. Enfatizam, ainda, que as equipes de saúde das UBS deveriam ser apoiadas para o acolhimento e o cuidado das pessoas em sofrimento mental, favorecendo o vínculo com a equipe de referência no território adscrito da UBS.

Ainda existem pontos importantes a serem desenvolvidos, como a atuação por meio das linhas de cuidado e a construção de fluxos e instrumentos que facilitem o diálogo entre os serviços. Os trabalhadores chamam a atenção para a contrarreferência de serviços como o Ciava, pois, como coordenador da atenção às vítimas do desastre, deveria estar trabalhando em coerência com a rede de atenção. Faz-se necessária a construção de uma RAS consistente e resolutiva, que facilite o acesso do usuário a serviços de qualidade e que permita a integralidade do cuidado.

\section{Considerações finais}

Considerando as dimensões do cenário que envolveu o desastre apresentado, compreender como o sistema de saúde se organizou para atender às demandas que surgiram se faz importante também para orientar situações semelhantes no futuro. Uma vez identificadas as fragilidades, elas podem ser corrigidas, a fim de evitar falhas semelhantes, e, no que tange aos pontos positivos, eles podem ser estimulados e promovidos.

A rede de serviços de saúde não estava preparada para a alta demanda que surgiu, exigindo uma organização abrupta e sem precedentes. Embora um desastre de tais proporções não encontre nenhum serviço preparado para seu enfrentamento, os desafios encontrados expuseram as fragilidades do sistema de saúde do município, assim como a falta de comunicação e de fluxos definidos entre os serviços. A falta de coordenação do processo de cuidado pela gestão municipal, principalmente no que se refere aos serviços de APS, demandou esforços das equipes para atuarem frente às vítimas, lidando com as dificuldades já existentes.

Apesar das dificuldades, pontos positivos puderam ser observados, como os serviços de saúde mental no município, que encontraram nesse período a oportunidade para consolidar suas práticas e expandir sua atuação, recebendo reconhecimento dos demais serviços. Também é resultado das necessidades decorrentes do desastre o estímulo à atuação multiprofissional, objetivando a integralidade do cuidado das vítimas. A atuação frente ao desastre encaminhou discussões para a construção de uma linha de cuidado, no entanto, é importante ressaltar que, não somente no caso das vítimas, mas das demais necessidades da população, essa discussão se faz necessária.

Ainda existem questões importantes a serem estimuladas, como a atuação através das linhas de cuidado e a construção de fluxos e instrumentos que facilitem o diálogo entre os serviços. Assim, faz-se necessária a construção de uma Rede de Atenção à Saúde consistente e resolutiva, que permita que o usuário tenha acesso a serviços de qualidade e que favoreçam a integralidade do cuidado. 


\section{Colaboradores}

Todos os autores contribuíram substancialmente para a concepção e o planejamento ou para a análise e a interpretação dos dados, bem como para a elaboração do rascunho ou revisão crítica do conteúdo e também participaram da aprovação da versão final do manuscrito.

Cristiane Wagner, Mariane Pergher Soares e Bruna Skrebsky realizaram a coleta e a análise dos dados, bem como a elaboração do texto do artigo. Beatriz Unfer e Taísa Gomes Ferreira realizaram a orientação e a supervisão da pesquisa, assim como revisão e aprovação dos textos.

\section{Referências}

BRASIL. Constituição da República Federativa do Brasil, de 1988. Diário Oficial [da] República Federativa do Brasil, Brasília, DF, 1988.

. Decreto n ${ }^{\circ} 7.508$, de 28 junho de 2011.

Regulamenta a Lei no 8.080, de 19 de setembro de 1990, para dispor sobre a organização do Sistema Único de Saúde - SUS, o planejamento da saúde, a assistência à saúde e a articulação interfederativa, e dá outras providências. Diário Oficial [da] República Federativa do Brasil, Brasília, DF, 2011.

. Ministério da Integração Nacional. Secretaria Nacional de Defesa Civil. Política Nacional de Defesa Civil. Brasília, DF: Ministério da Integração, 2007. Disponível em: <http://www.mi.gov.br/c/document_library/get_file?uuid=6aa2e891-98f6-48a6-8f47147552c57f94\&groupId=10157>. Acesso em: 10 ago. 2017.

. Ministério da Saúde. DATASUS. c2017.

Disponível em: <http://datasus.saude.gov.br/cadastro-nacional-de-estabelecimentos-de-saude $>$. Acesso em: 3 ago. 2017.

Ministério da Saúde. Secretaria de Atenção à Saúde. Departamento de Atenção Básica. Histórico de cobertura da saúde da família. Brasília, DF, 2012a. Disponível em: <http://dab.saude.gov.br/dab/historico_cobertura_sf/historico_cobertura_sf_relatorio.php >. Acesso em: 20 dez. 2015. Ministério da Saúde. Secretaria de Atenção à Saúde. Departamento de Atenção Básica. Política Nacional de Atenção Básica. Brasília, DF: Ministério da Saúde, 2012b. Disponível em: <http://189.28.128.100/ dab/docs/publicacoes/geral/pnab.pdf $>$. Acesso em: 10 ago. 2017.

CARMO E. H.; PENNA, G.; OLIVEIRA, W. K. Emergências de saúde pública: conceito, caracterização, preparação e resposta. Estudos Avançados, São Paulo, v. 22, n. 64, dez. 2008. Disponível em: <http:// www.scielo.br/scielo.php?script=sci_arttext\&pid $=$ S0103-40142008000300003 $>$. Acesso em: 10 ago. 2017.

\section{INSTITUTO BRASILEIRO DE GEOGRAFIA E} ESTATÍSTICA (IBGE). Censo Demográfico 2010. Censo Demográfico, Rio de Janeiro, 2010. Disponível em: <https://biblioteca.ibge.gov.br/visualizacao/periodicos/552/cd_2010_agsn_if.pdf $>$. Acesso em: 20 dez. 2015. 
KIPPER, L.; DA'LAGNOL, M. M.; PAULESKI, L. C. Mutirão de atendimento às vítimas do incêndio em boate de Santa Maria no CIAVA/HUSM: perfil epidemiológico. In: JORNADA ACADÊMICA INTEGRADA, Santa Maria. Anais... Santa Maria: [S. n.], 2014.

Disponível em: <https://portal.ufsm.br/jai/anais/trabalho.html?action=anais>. Acesso em: 18 jun. 2016.

MENDES, E. V. As redes de atenção à saúde. Ciência \&t Saúde Coletiva, Rio de Janeiro, v. 15, n. 5, p. 2297-2305, ago. 2010. Disponível em: <http:// www.scielo.br/scielo.php?script=sci_arttext\&pid $=$ S1413-81232010000500005 $>$. Acesso em: 10 ago. 2017.

MINAYO, M. C. S. O desafio do conhecimento: metodologia de pesquisa social em saúde. São Paulo: Hucitec, 2010.

SÃO PAULO. Secretaria de Estado da Saúde. Desastres e incidentes com múltiplas vítimas: Plano de Atendimento: Preparação Hospitalar. São Paulo: Secretaria de Saúde, 2012. Disponível em: <http:// www.saude.sp.gov.br/resources/ses/perfil/gestor/ homepage/desastres/preparacao-hospitalar-para-atendimento-de-desastres-e-incidentes-com-multiplas-vitimas/incidentes_com_multiplas_vitimas_e_desastres_2012.pdf>. Acesso em: 20 dez. 2015.
SANTA MARIA. Prefeitura Municipal de Santa Maria. Agência de Desenvolvimento de Santa Maria. Santa Maria. Revista Santa Maria, Santa Maria, 2011. Disponível em: <http://adesm.org.br/wp-content/uploads/2011/11/Revista_Santa_Maria-4ed-port-ing-bx. pdf $>$. Acesso em: 9 fev. 2014.

Prefeitura Santa Maria. Secretaria de Município da Saúde. c2017. Disponível em: <http://www.santamaria.rs.gov.br/saude/>. Acesso em: 9 fev. 2017.

STARFIELD, B. Atenção primária: equilíbrio entre necessidades de saúde, serviços e tecnologia. Brasília, DF: Ministério da Saúde, 2002.

THIOLLENT, M. Metodologia da Pesquisa-ação. 14. ed. São Paulo: Cortez, 2005.

TRIPP, D. Pesquisa-ação: uma introdução metodológica. Educação e Pesquisa, São Paulo, v. 31, n. 3, p. 443-466, 2005. Disponível em: $<$ http://www.scielo.br/scielo.php?pid=S1517$-97022005000300009 \&$ script $=$ sci_abstract\&tlng=pt>. Acesso em: 9 fev. 2017.

Recebido para publicação em maio de 2017

Versão final em setembro de 2017

Conflito de interesses: inexistente

Suporte financeiro: não houve 